\title{
A Hybrid Tuned Mass Damper for Response Mitigation of Offshore Platforms
}

Quoc Huong Cao ( $\sim$ huongcq81@gmail.com )

Thang Long Urban Development and Construction JSC, Hanoi, Vietnam https://orcid.org/0000-00026352-3787

\section{Research Article}

Keywords: Tuned mass damper, Hybrid tuned mass damper, Passive damper, Vibration control, Multiobjective Optimization

Posted Date: February 11th, 2022

DOI: https://doi.org/10.21203/rs.3.rs-1230815/v2

License: (1) This work is licensed under a Creative Commons Attribution 4.0 International License. Read Full License 


\title{
A HYBRID TUNED MASS DAMPER \\ FOR RESPONSE MITIGATION OF OFFSHORE PLATFORMS
}

\author{
Quoc Huong Cao ${ }^{1}$ \\ ${ }^{1}$ Thang Long Urban Development and Construction JSC, Hanoi, Vietnam
}

Corresponding author: Quoc Huong Cao

E-mail address: huongcq81@gmail.com

ORCID: 0000-0002-6352-3787

\begin{abstract}
A new hybrid type of the Tuned Mass Damper (HTMD), which consists of a Tuned Liquid Column Damper (TLCD) fixed on the top of a traditional Tuned Mass Damper (TMD), is developed for vibration control of an offshore platform. The results obtained from the parametric investigation show that the mass ratio between TLCD and TMD significantly affects the HTMD's performance. To assess the effectiveness and robustness of HTMD, extensive comparisons are made between an optimized HTMD and an optimum TMD with the same weight as the HTMD. The numerical computations indicate that the proposed HTMD offers a higher level of effectiveness in suppressing structural vibrations compared with a traditional TMD. However, the optimum HTMD is not robust in resisting the variation of the structural stiffness.
\end{abstract}

Keywords: Tuned mass damper; Hybrid tuned mass damper; Passive damper; Vibration control; Multiobjective Optimization

\section{INTRODUCTION}

An offshore platform is a crucial structure for projects of exploitation of ocean oil and gas. It is usually built in deep-water areas from a few meters to hundreds of meters. Platforms located in ocean 
environments are always affected by very harsh environmental conditions such as floating ice, storm, water waves and earthquake (Ou et al., 2007; Wu et al., 2018; Wu et al., 2016; Jafarabad et al., 2014). Different causes lead to platforms' vibration, including oscillations induced by natural conditions as mentioned above and other operating conditions. In the case that the platform vibration has a large amplitude, it may cause severe harm to the structures (Ou et al., 2007; Wu et al., 2018; Wu et al., 2016) as well as discomfort to human occupants on it (Ou et al., 2007; Wu et al., 2018). Therefore, it is necessary to find a suitable and effective solution for suppressing vibrations of offshore platforms.

Many studies have been conducted to control harmful vibrations in the platform structures. Two leading solutions for this problem are (i) to equip a damping isolation system for the platform (Ou et al., 2007), and (ii) to add external damping devices to the primary structure of the platforms (Wu et al., 2018; Wu et al., 2016; Xue et al., 2016; Jafarabad et al., 2014). For instance, Ou et al. (2007) developed a damping isolation system with rubber bearings and viscous dampers for the JZ20-2MUQ offshore platform's vibration control. Meanwhile, to mitigate the seismic vibration of offshore platforms, Jafarabad et al. (2014) proposed a hybrid damping system that consists of a friction damper device (FDD) and a tuned mass damper (TMD). Wu et al. (2018) used a TMD with the nonlinear magnetic force for an offshore platform, while Xue et al. (2016) evaluated the effectiveness and the robustness of a Pounding Tuned Mass Damper through the experimental study.

Different types of TMD (including TLCD) have been investigated and applied widely and they had been proven to be simple and effective dampers (Chang and Hsu, 1998; Gao et al., 1999; Samali et al., 2004; Wu et al., 2005; Li and Zhu, 2006; Diana et al., 2013; Momtaz et al., 2017; Sun et al., 2018; Vellar et al., 2019). Thus, using one or more TMDs for reducing undesired vibration is still a suitable strategy, and this strategy has also attracted attention from many researchers. In particular, researchers have proposed and developed various modified types of TMD to enhance the performance and robustness of TMD. For example, Li and Zhu (2006) developed a Double Tuned Mass Dampers (DTMD) consisting of one smaller TMD coupled with one larger TMD. A tuned viscous mass damper (VMD) has been proposed by Ikago et al. (2012). Zhang et al. (2013) studied a pounding tuned mass 
damper (PTMD). PTMD is considered a combination of conventional TMD and pounding effects. Jafarabad et al. (2014) also developed a hybrid damping system. This hybrid system is composed of a tuned mass damper (TMD) fixed on a friction damper device (FDD) to reduce the seismic vibrations of structures. For structural vibrations at low frequencies, a modified version of the traditional TLCD is proposed by Al-Saif et al. (2011). In this improved TLCD, the orifice in TLCD is replaced by a steel ball, and it is named a tuned liquid column ball damper (TLCBD). This study showed that the ball-tube diameter ratio significantly influences the vibration suppression capacity of TLCBD. Cao (2021) developed a hybrid vibration absorber which is combined by a tuned liquid column damper (TLCD) and an undamped TMD (Cao, 2021). The results obtained from the above works have indicated that upgraded TMD systems are better than the traditional ones.

To further enhance the effectiveness of TMD, a new hybrid type of TMD (HTMD) for reducing an offshore platform's vibrations is proposed in this study. This hybrid device is composed of a TLCD fixed on a traditional TMD with a mass ratio unchanged. Unlike active or semi-active dampers, this damper does not depend on an external power source and a well-designed control algorithm for its operation. Furthermore, it is also easy to install and adjust, cost-effective and low maintenance requirements.

The paper is organized as follows. After the introduction, Section 2 presents the structural modelling of the HTMD-Platform system. Section 3 includes a study on the influence of HTMD's parameters in reducing structural vibrations and design optimization. In the optimum design, Genetic Algorithms are chosen to find the optimal parameters of HTMD through solving optimization problems. Based on results obtained in Section 3, the performance and robustness of HTMD in mitigating the platform response is determined and compared with an optimum TMD in Section 4. Then the multi-objective optimal design is mentioned in Section 5. Finally, conclusions drawn from this research is shown in Section 6. 


\section{MATHEMATICAL MODEL}

\subsection{Modelling of a platform}

Let us analyse an existing offshore platform subjected to external force as Fig. 1. The structural system considered is a steel jacket offshore platform JZ20-2MUQ (Ou et al., 2007; Xue et al., 2016) in the Bohai Sea, China. Fig. 1 shows the real model and the idealised model of the platform. Key parameters of the platform are presented in Table 1. In this study, the horizontal vibration of the platform only is of interest (assume that its vertical and rotational vibrations are negligible). Here, there are several the following assumptions:

a) For the sake of vibration analysis, the offshore platform is idealized as an equivalent system with a single degree of freedom (SDOF).

b) The external load $F_{(t)}$ acting on the platform is a harmonic excitation with the frequency $\Omega$

$$
F_{(t)}=F_{0} \sin (\Omega t)
$$

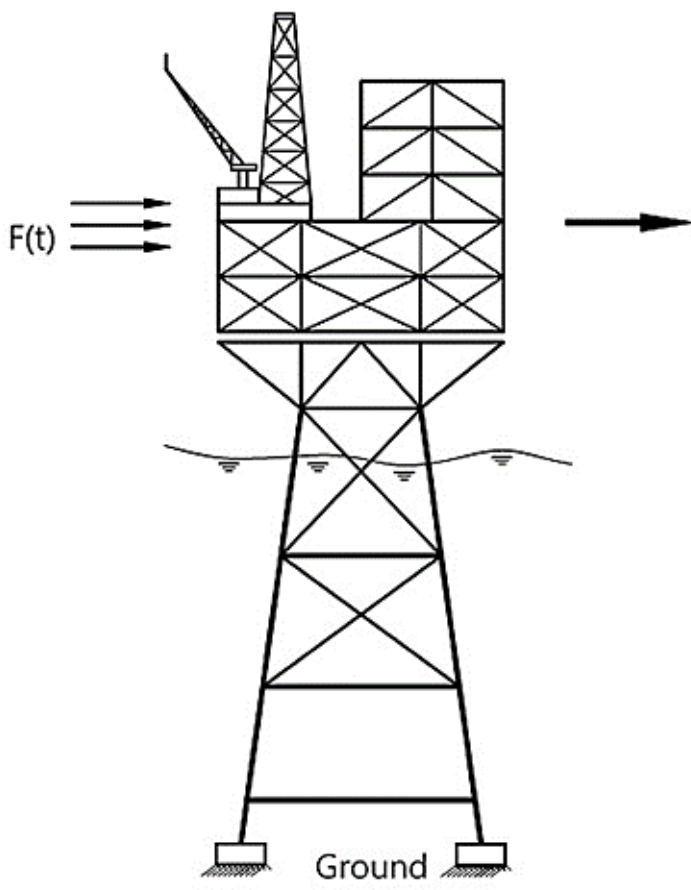

a)

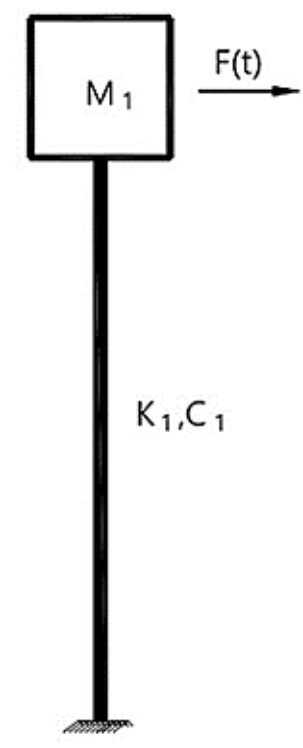

b)

Fig. 1. Offshore platform model

a) JZ20-2MUQ platform model, b) Idealized model 


\subsection{HTMD-Platform system}

A calculation model of a SDOF platform equipped with an HTMD under external excitation is simplified as Fig. 2. The HTMD consists of a single TLCD fixed on a traditional TMD with a constant mass ratio. The TLCD is assumed to be a uniform U-shaped liquid column with a cross-sectional area $A$, a head-loss coefficient $\zeta$, a total length $L$, a horizontal length $B$, and the liquid density $\rho$. The TMD's parameters include the mass $M_{2}$, the stiffness $K_{2}$ and the damping $C_{2}$. Note that the mass of TMD included the mass of the container which contains the liquid. The platform has the mass $M_{l}$, the stiffness $K_{1}$, and the damping $C_{1}$. Let us assume that $U_{1}$ is the displacement of the platform; $U_{2}$ is the relative displacement of the TMD mass; and $U_{3}$ is the vertical displacement of the liquid column.

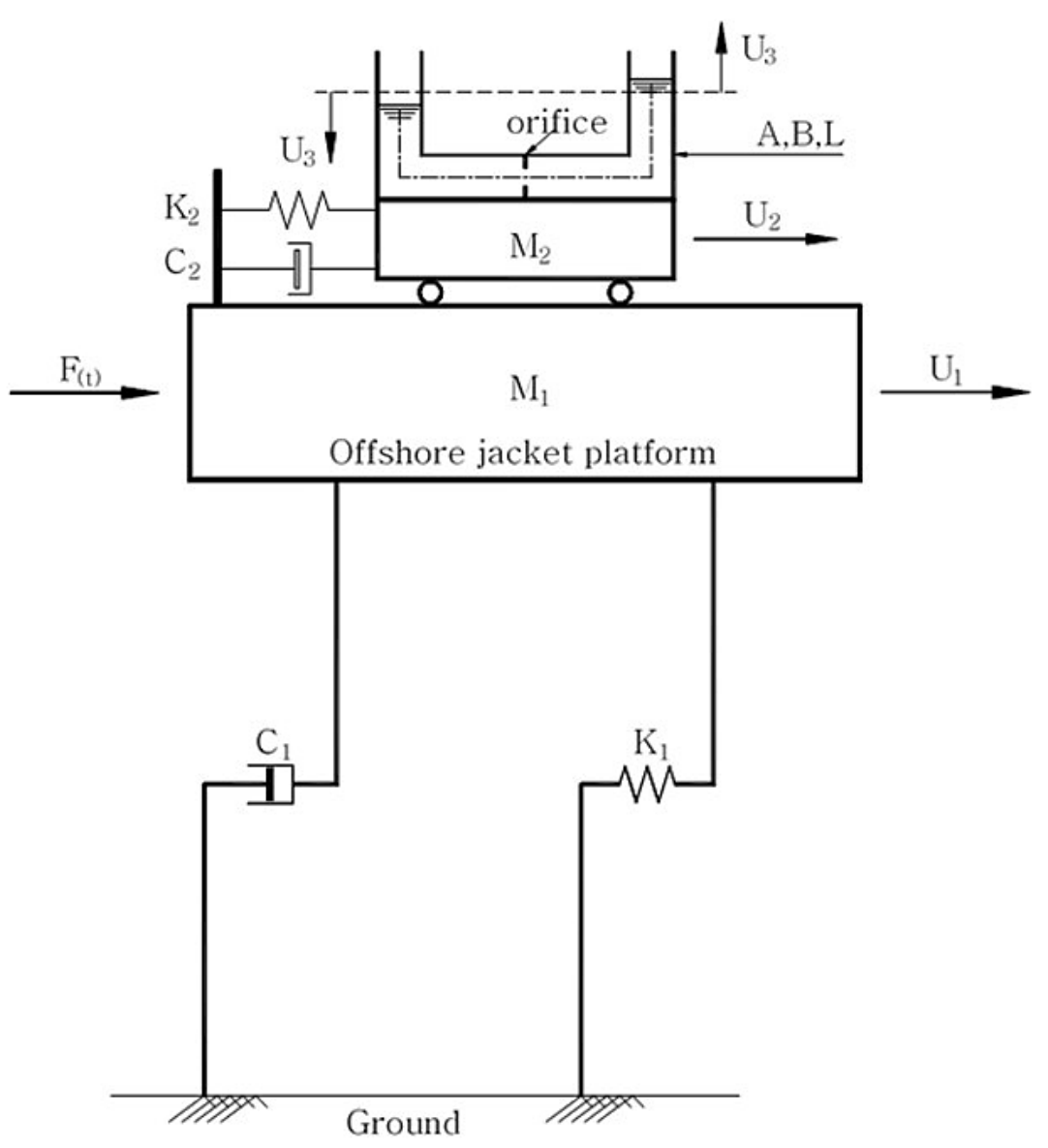

Fig. 2. Calculation model of the HTMD-Platform system 
The equation of liquid column relative motion can be described as:

$\rho A L \ddot{U}_{3}+\frac{1}{2} \rho A \zeta\left|\dot{U}_{3}\right| \dot{U}_{3}+2 \rho A g U_{3}=-\rho A B\left(\ddot{U}_{1}+\ddot{U}_{2}\right) \quad\left(\left|\ddot{U}_{3}\right| \leq \frac{L-B}{2}\right)$.

In Eq. (2), $\dot{U}_{3}$ and $\ddot{U}_{3}$ denote the first and second derivatives of $U_{3}$ with respect to time, respectively; and $g$ is the gravity acceleration. Denoting the natural frequency of liquid motion $\left(\omega_{d}\right)$, mass $\left(m_{d}\right)$ and length ratio of liquid column $(\gamma)$ as:

$\omega_{d}=\sqrt{\frac{2 g}{L}}$,

$m_{d}=\rho A L$

and

$\gamma=\frac{B}{L}$

Note that the damping term $\frac{1}{2} \rho A \zeta\left|\dot{U}_{3}\right|$ in Eq. (2) is nonlinear. Let us assume that the sloshing phenomenon on the liquid surface is not significant and the motion amplitude of the liquid column is not too large. Hence, the damping of TLCD can be linearized. According to Gao and Kwok (1997), Yalla and Kareem (2000), with an assumed damping ratio $\xi_{d}$, the equivalent damping coefficient $c_{e q}$ is given by

$c_{e q}=2 m_{d} \xi_{d} \omega_{d}$

As a result, Eq. (2) can be simplified as

$\ddot{U}_{3}+2 \xi_{d} \omega_{d} \dot{U}_{3}+\omega_{d}^{2} U_{3}=-\gamma\left(\ddot{U}_{1}+\ddot{U}_{2}\right)$.

\section{b) Equations of motion of TMD mass}

The equation of relative motion of mass $M_{2}$ is expressed as

$\left(m_{d}+M_{2}\right) \ddot{U}_{2}+C_{2} \dot{U}_{2}+K_{2} U_{2}=-\left(m_{d}+M_{2}\right) \ddot{U}_{1}-\gamma m_{d} \ddot{U}_{3}$.

The natural frequency and damping ratio of TMD are expressed as follows: 
$\omega_{2}=\sqrt{\frac{K_{2}}{M_{2}}}$

and

$\xi_{2}=\frac{C_{2}}{2 M_{2} \omega_{2}}$

Eq. (8), can be rewritten as

$\left(\frac{m_{d}}{M_{2}}+1\right) \ddot{U}_{2}+2 \xi_{2} \omega_{2} \dot{U}_{2}+\omega_{2}^{2} U_{2}=-\left(\frac{m_{d}}{M_{2}}+1\right) \ddot{U}_{1}-\gamma \frac{m_{d}}{M_{2}} \ddot{U}_{3}$.

c) Equations of motion of Platform

The equilibrium equation of the offshore platform is determined as

$M_{1} \ddot{U}_{1}+C_{1} \dot{U}_{1}+K_{1} U_{1}-C_{2} \dot{U}_{2}-K_{2} U_{2}=F_{(t)}$.

Let us introduce the natural frequency and damping ratio of the platform as

$\omega_{1}=\sqrt{\frac{K_{1}}{M_{1}}}$

and

$\xi_{1}=\frac{C_{1}}{2 M_{1} \omega_{1}}$.

Eq. (12) is simplified as

$\ddot{U}_{1}+2 \xi_{1} \omega_{1} \dot{U}_{1}+\omega_{1}^{2} U_{1}-2\left(\frac{M_{2}}{M_{1}}\right) \xi_{2} \omega_{2} \dot{U}_{2}-\frac{M_{2}}{M_{1}} \omega_{2}^{2} U_{2}=\frac{F_{(t)}}{M_{1}}$.

d) Equations of motion of HTMD-Platform system

By combining Eqs. (1), (7), (11) and (15), the equations of motion for HTMD-Platform system are written in matrix form:

$\mathbf{M U ̈}+\mathbf{C} \dot{U}+\mathbf{K U}=\mathbf{F}$

in which:

$\mathbf{M}=\left[\begin{array}{ccc}1 & 0 & 0 \\ 1+\frac{m_{d}}{M_{2}} & 1+\frac{m_{d}}{M_{2}} & \gamma \frac{m_{d}}{M_{2}} \\ \gamma & \gamma & 1\end{array}\right]$ 
$\mathbf{C}=\left[\begin{array}{ccc}2 \xi_{1} \omega_{1} & -2\left(\frac{M_{2}}{M_{1}}\right) \xi_{2} \omega_{2} & 0 \\ 0 & 2 \xi_{2} \omega_{2} & 0 \\ 0 & 0 & 2 \xi_{d} \omega_{d}\end{array}\right]$

$\mathbf{K}=\left[\begin{array}{ccc}\omega_{1}^{2} & -\left(\frac{M_{2}}{M_{1}}\right) \omega_{2}^{2} & 0 \\ 0 & \omega_{2}^{2} & 0 \\ 0 & 0 & \omega_{d}^{2}\end{array}\right]$

$\mathbf{F}=\left[\begin{array}{c}\left(F_{0} / M_{1}\right) \sin (\Omega t) \\ 0 \\ 0\end{array}\right]$

$\mathbf{U}=\left[\begin{array}{l}U_{1} \\ U_{2} \\ U_{3}\end{array}\right]$

$\dot{\mathbf{U}}=\left[\begin{array}{c}\dot{U}_{1} \\ \dot{U}_{2} \\ \dot{U}_{3}\end{array}\right]$

$\ddot{\mathbf{U}}=\left[\begin{array}{c}\ddot{U}_{1} \\ \ddot{U}_{2} \\ \ddot{U}_{3}\end{array}\right]$

The following nondimensional quantities are introduced to simplify the equations of motion governing HTMD-Platform system.

The mass ratio of the TLCD is

$\mu_{1}=\frac{m_{d}}{M_{1}}$

The mass ratio of the TMD is

$\mu_{2}=\frac{M_{2}}{M_{1}}$.

The mass ratio of the HTMD is

$\mu=\mu_{1}+\mu_{2}=\frac{m_{d}+M_{2}}{M_{1}}$.

The mass ratio between TLCD and TMD in an HTMD is

$\mu_{3}=\frac{\mu_{1}}{\mu_{2}}=\frac{m_{d}}{M_{2}}$.

Thus, Eqs. (16a), (16b) and (16c) can be rewritten as 
$\mathbf{M}=\left[\begin{array}{ccc}1 & 0 & 0 \\ 1+\mu_{3} & 1+\mu_{3} & \gamma \mu_{3} \\ \gamma & \gamma & 1\end{array}\right]$

$\mathbf{C}=\left[\begin{array}{ccc}2 \xi_{1} \omega_{1} & -2 \mu_{2} \xi_{2} \omega_{2} & 0 \\ 0 & 2 \xi_{2} \omega_{2} & 0 \\ 0 & 0 & 2 \xi_{d} \omega_{d}\end{array}\right]$

$\mathbf{K}=\left[\begin{array}{ccc}\omega_{1}^{2} & -\mu_{2} \omega_{2}^{2} & 0 \\ 0 & \omega_{2}^{2} & 0 \\ 0 & 0 & \omega_{d}^{2}\end{array}\right]$

\subsection{Dynamic magnification factor of structural response}

According to Gil-Martín et al. (2012), the steady-state dynamic magnification factor (DMF) of the structural response can be determined as

$D M F=\frac{M a x U_{1}}{\left(F_{0} / K_{1}\right)}$

In this study, let the frequency ratio as

$\alpha=\frac{\Omega}{\omega_{1}}$.

The tuning ratios $\left(\beta_{1}, \beta_{2}\right)$, for both TLCD and TMD, can be denoted as

$\left\{\begin{array}{l}\beta_{1}=\frac{\omega_{d}}{\omega_{1}} \\ \beta_{2}=\frac{\omega_{2}}{\omega_{1}}\end{array}\right.$

Hence, the tuning ratio of HTMD is

$\beta=\frac{\omega_{d}}{\omega_{2}}=\frac{\beta_{1}}{\beta_{2}}$.

The platform response is the function in which $\alpha, \beta_{1}, \beta_{2}, \xi_{1}, \xi_{2}, \xi_{d}, \mu_{1}$ and $\mu_{2}$ are variables. It is noted that the values of $\mu_{1}$ and $\mu_{2}$ can be replaced by $\mu$ and $\mu_{3}$ through Eqs. (17c) and (17d). With each structure, two parameters $\xi_{1}$ and $\alpha$ are determined. The remaining parameters will be discussed through a parametric study in the next section. 


\section{OPTIMUM DESIGN}

\subsection{Parametric investigation}

A parametric study involving the effects of the frequency range, mass ratios, and damping ratios on the HTMD's performance will be discussed here. The input parameters of the platform are shown in Table 1. Let us assume that the excitation frequency domain considered corresponds to $0.5 \leq \alpha \leq$ 1.5 and the harmonic force's magnitude is $1.0 \times 10^{6}(\mathrm{~N})$.

Table 1. The parameters of JZ20-2MUQ platform

\begin{tabular}{lll}
\hline Parameter & Symbol & Value \\
\hline The mass of the platform & $M_{1}$ & $3.127 \times 10^{6} \mathrm{~kg}$ \\
The stiffness of the platform & $K_{l}$ & $9.344 \times 10^{6} \mathrm{~kg} / \mathrm{s}^{2}$ \\
The damping coefficient of the platform & $C_{l}$ & $1.367 \times 10^{5} \mathrm{~kg} / \mathrm{s}$ \\
The natural frequency of the platform & $\omega_{1}$ & $5.4663 \mathrm{rad} / \mathrm{s}$ \\
The damping ratio of the platform & $\xi_{1}$ & 0.04 \\
\hline
\end{tabular}

a) Effects of the tuning ratios $\beta, \beta_{1}$

It is assumed that the tuning ratio of $\operatorname{HTMD}(\beta)$ is considered in the range of $[0.5,1.5]$ and the mass ratios are 0.03 and 0.1 for $\mu_{3}$ and $\mu$, respectively. Here, $D M F_{\max }$ is denoted as the peak dynamic magnification factor of the platform response in the excitation frequency range of $\left[\Omega_{1}, \Omega_{2}\right]$. The influence of the tuning ratio $\beta$ on the $D M F_{\max }$ curve is shown in Fig. 3 for different values of $\beta_{1}$. It is noted that the lowest point of $D M F_{\max }$ curve (corresponding to each $\beta_{1}$ ) also represents the capacity of HTMD in absorbing vibration. This means that the most effective HTMD will has the $D M F_{\max }$ curve with the lowest bottom. Based on the lowest point of each $D M F_{\max }$ curve in Fig. 3, some conclusions are drawn as follows: 
(i) There exists an optimum value of $\beta$ for each $\beta_{1}$ given. For example, in the case of $\beta_{1}=$ 0.9 , the optimum value of $\beta$ is 0.97 .

(ii) The value of the lowest point on $D M F_{\max }$ curve tends to increase if the value of $\beta_{1}$ rises.

(iii) There is an uptrend in the optimal value of $\beta$ as $\beta_{1}$ increases (the exception of $\beta_{1}=1.0$ ). In the figure, the optimal value of $\beta$ grows from 0.83 to 1.14 when $\beta_{1}$ runs from 0.8 to 1.2. It is noted that the system happens the resonance at $\beta_{1}=1\left(\omega_{d}=\omega_{1}\right)$. Thus, the behaviour of system in this case is different from remaining cases of $\beta_{1}$.

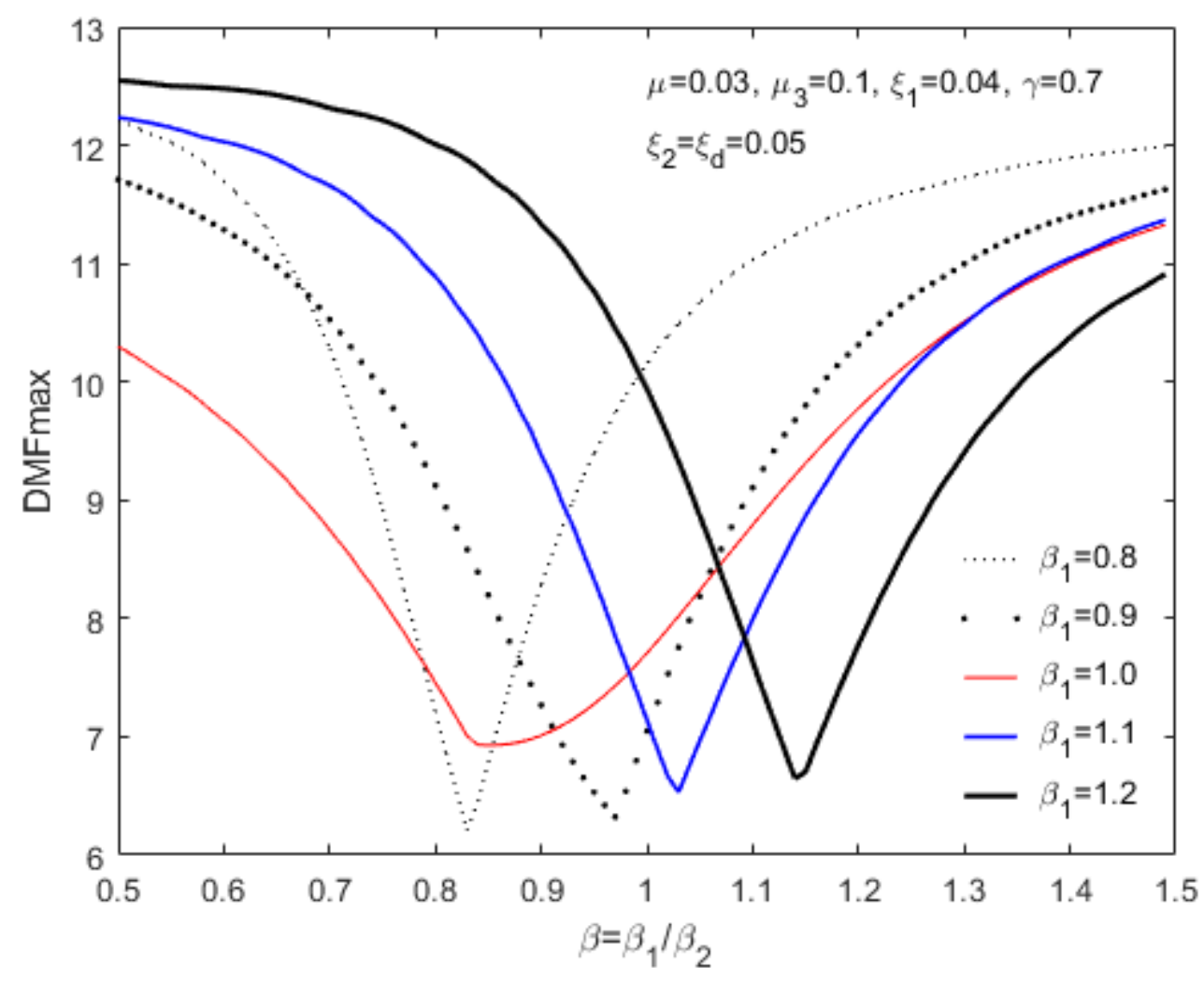

Fig. 3. $D M F_{\max }$ curves for different values of $\beta_{1}$

\section{b) Effects of the damping ratios $\xi_{2}, \xi_{d}$}

Fig. 4 and Fig. 5 depict $D M F_{\max }$ curves of the platform response for five different values of the damping ratio $\xi_{2}$ and for five different values of the damping ratio $\xi_{d}$, respectively. The tuning ratio $\beta_{1}$ selected is 0.9 for both Fig. 4 and Fig. 5. The damping ratio $\xi_{d}$ is 0.05 for the cases in Fig. 4 , and $\xi_{2}=0.1$ for the cases in Fig. 5. Obviously, the performance of HTMD reduces when the damping 
ratio $\xi_{2}$ rises from 0.05 to 0.2 in Fig. 4. Meanwhile, the vibration absorption capacity of HTMD increases if the damping ratio $\xi_{\mathrm{d}}$ goes from 0.01 to 0.2 in Fig. 5.

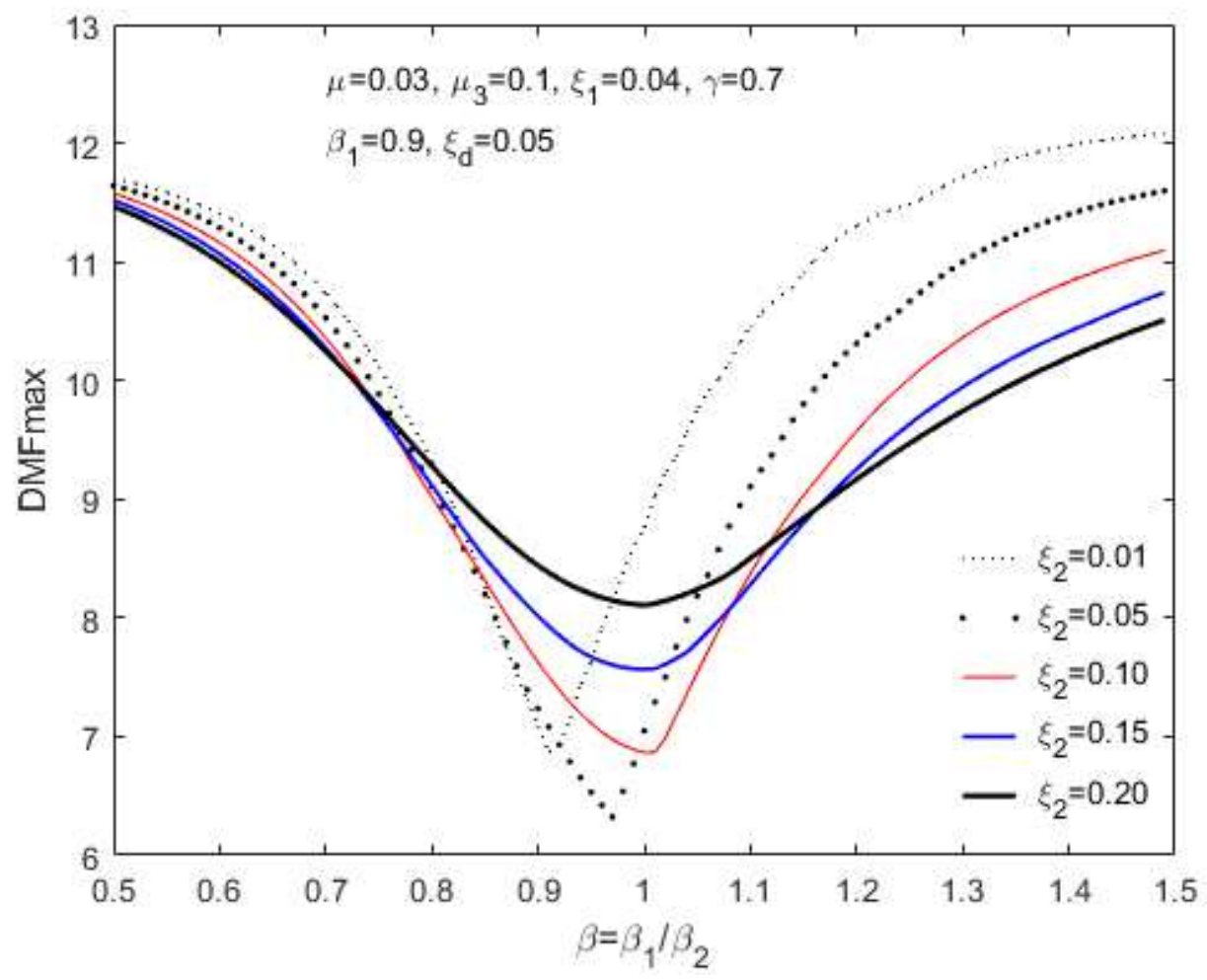

Fig. 4. $D M F_{\max }$ curves for different values of $\xi_{2}$

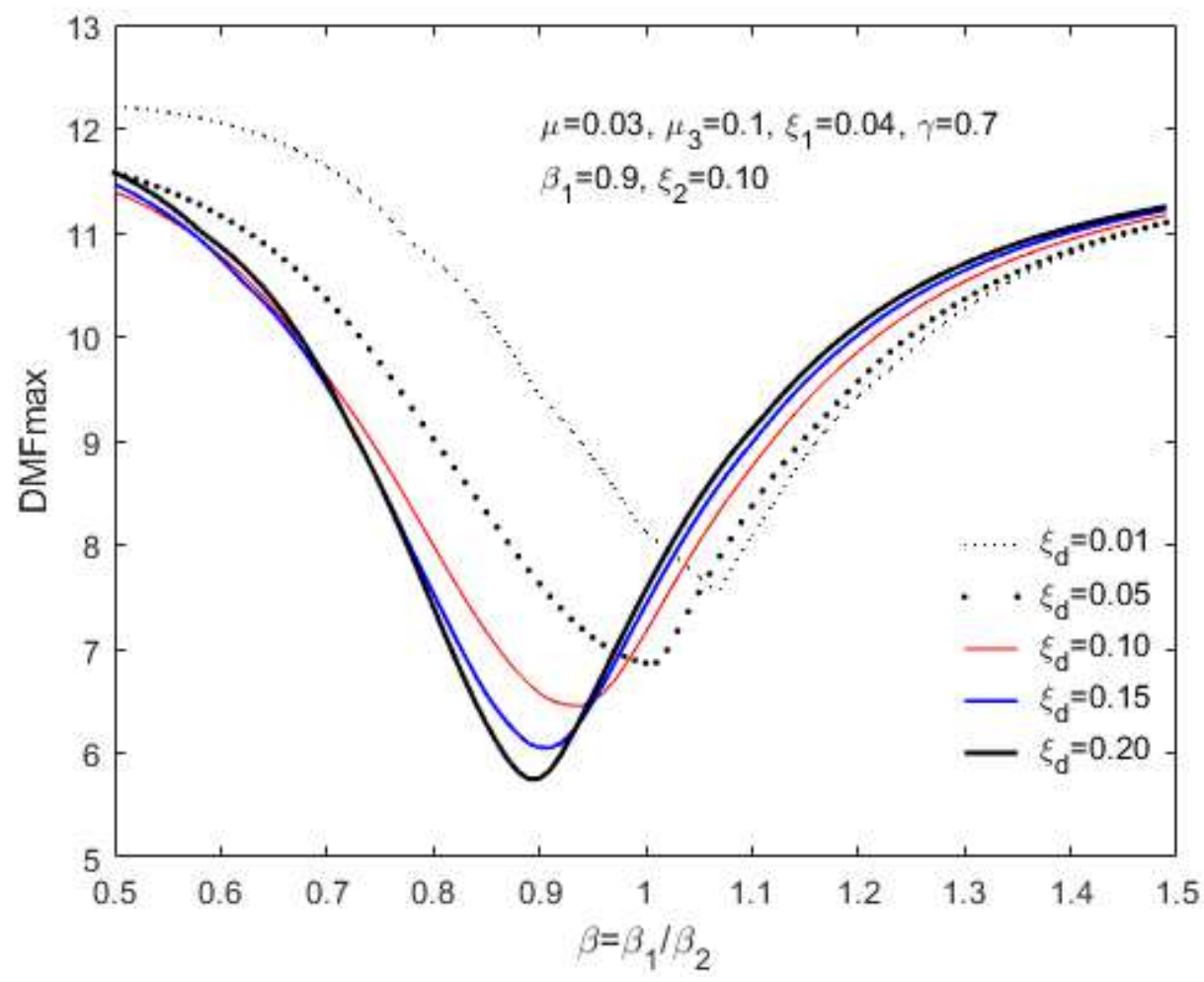

Fig. 5. $D M F_{\max }$ curves for different values of $\xi_{d}$ 
c) Effects of the mass ratios $\mu, \mu_{3}$

Fig. 6 shows $D M F_{\max }$ curves with three different values of the mass ratio $(\mu=1 \%, 3 \%$ and $5 \%)$ while the ratio $\mu_{1} / \mu_{2}$ varies in the range of $[0,1]$. The length ratio of liquid column is fixed to be 0.7. With the assumption that the tuning ratios are set as $\beta_{1}=\beta_{2}=1$ and the damping ratios are chosen as $\xi_{2}=\xi_{d}=0.05$. Obviously, the vibration suppression performance of HTMD increases as the mass ratio $\mu$ increases. In addition, there are two points highlighted in Fig. 6 as follows:

(i) It is possible to determine the optimum value of $\mu_{3}$ for each $\mu$ chosen.

(ii) The optimum value of $\mu_{3}$ will get larger if $\mu$ continues to increase. For instance, the optimum value of $\mu_{3}$ is obtained as 0.04 with $\mu=0.03$ and the optimum value of $\mu_{3}$ is found as approximately 0.07 as $\mu=0.05$.

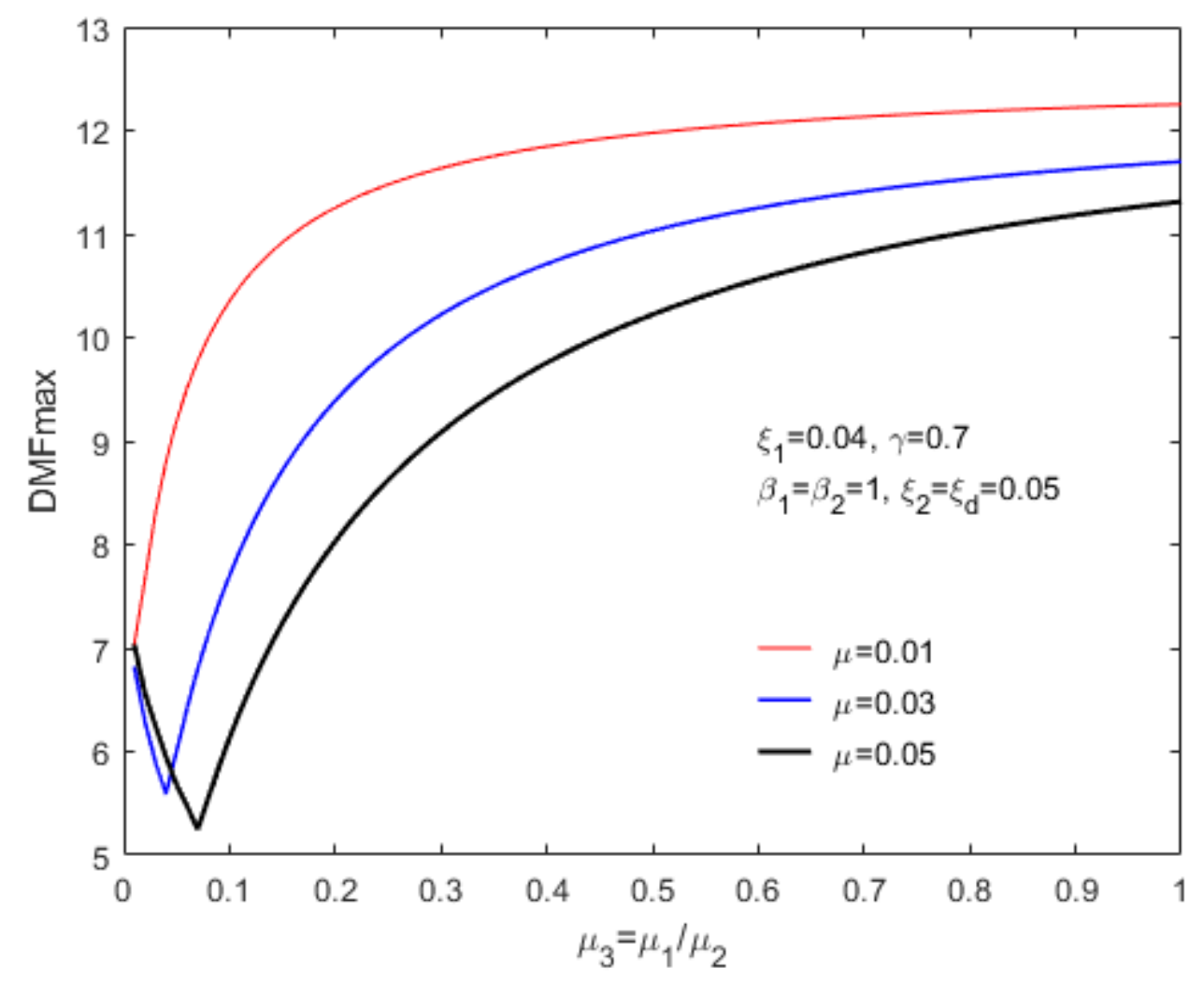

Fig. 6. $D M F_{\max }$ curves for different values of $\mu$ 
Fig. 7 describes the frequency response curves of the platform for different values of $\mu_{3}$ (or $\left.\mu_{1} / \mu_{2}\right)$. In this calculation, a mass ratio $(\mu)$ is chosen to be 0.03 . It can be observed in Fig. 7 , the platform response is transformed from a three-peak characteristic (if $\mu_{3}$ increases from $1 / 30$ to $1 / 20$ ) to a twopeak characteristic (if $\mu_{3}$ is from $1 / 10$ to 1.0 ) or a one-peak characteristic (if $\mu_{3}=10$ ). Obviously, the importance of choosing $\mu_{3}$ can be stated as follows:

(i) The response mitigation effectiveness of HTMD is significant as the ratio $\mu_{3}<1$.

(ii) The capacity of HTMD in suppressing vibration is less significant if $\mu_{3} \geq 1$.

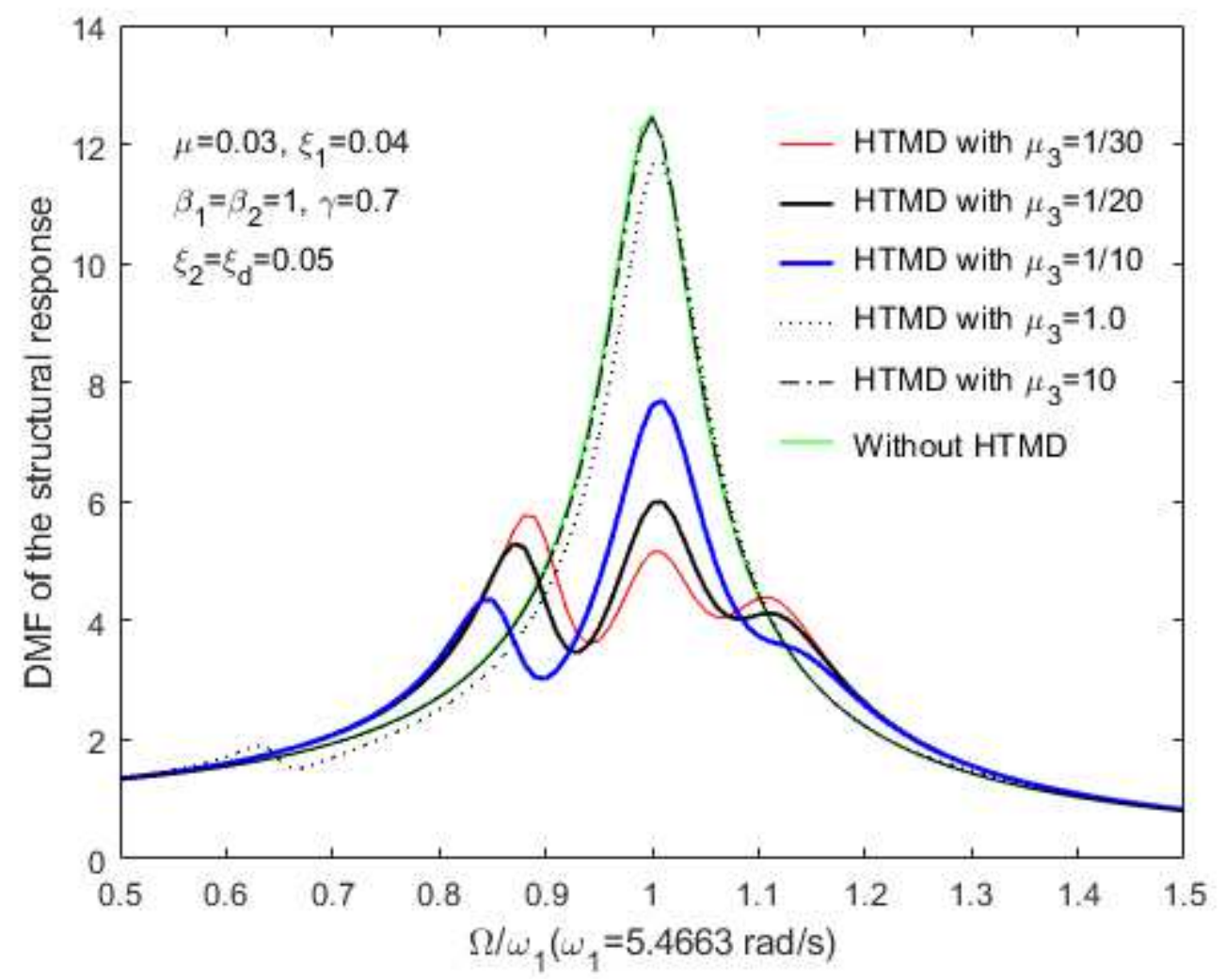

Fig. 7. Frequency response curves of the platform in different cases of $\mu_{3}$

As discussed above, it is possible to conclude that there exists a set of proper values of $\beta_{1}, \beta_{2}, \xi_{2}, \xi_{d}, \mu$, and $\mu_{3}$ for maximizing the efficiency of an HTMD in absorbing platform vibrations. 


\subsection{Optimum parameters}

The maximum capacity of HTMD in suppressing vibration will be achieved if the HTMD's design parameters reach optimal values. Nevertheless, finding a closed-form solution for all optimum parameters of HTMD is not easy. This means that the optimum parameters of HTMD should be determined through optimization tools.

The target of the parametric optimization is to maximize the performance of HTMD. This means that $D M F_{\max }$ of the platform response when the excitation frequency changes in the domain of $\left[\Omega_{1}\right.$, $\Omega_{2}$ ] must be minimized. By combining Eq. (20), the objective function $(O b j)$ for $D M F_{\max }$ of the platform response can be expressed as

$$
O b j=\operatorname{Min}\left\{\left.D M F_{\max }\right|_{\alpha_{2}} ^{\alpha_{1}}\right\}
$$

In Eq. (23), $\alpha_{1}$ and $\alpha_{2}$ are the upper and lower limits of the frequency ratio corresponding to $\Omega_{1}$ and $\Omega_{2}$. Here, Eq. (23) is a complex function with six variables, including the mass ratios $\left(\mu, \mu_{3}\right)$, the tuning ratios $\left(\beta_{1}, \beta_{2}\right)$ and the damping ratios $\left(\xi_{2}, \xi_{d}\right)$. Hence, choosing a suitable optimization algorithm is needed for this objective function.

Genetic Algorithm (GA) is a powerful solution-search or optimization technique based on a natural selection process (McCall 2005). Although there are more robust optimization algorithms, GA is still an effective optimization technique (McCall, 2005; Ahadi et al., 2012) to solve complex optimization problems including unconstrained or constrained optimization problems with many variables. In addition, GA is also a good solution for problems that the objective function is stochastic, discontinuous, or non-linear. Therefore, the Genetic Algorithm (GA) is chosen for solving optimization problems in this research.

A genetic algorithm operates on a population of artificial chromosomes. It is started by a randomly created population of chromosomes. GA conducts a process of evaluation and selection based on their relative fitness to produce the next generation. Good parent chromosomes are selected during 
recombination. Then genetic material from these parents is recombined to produce child chromosomes. The process is iterated, a series of the next generations develop, and the average fitness of the chromosomes tends to be better until meeting one of the stopping conditions. Finally, the algorithm reports the best chromosome, which is also the best solution to a given problem. Fig. 8 describes a flowchart of the GA optimization process.

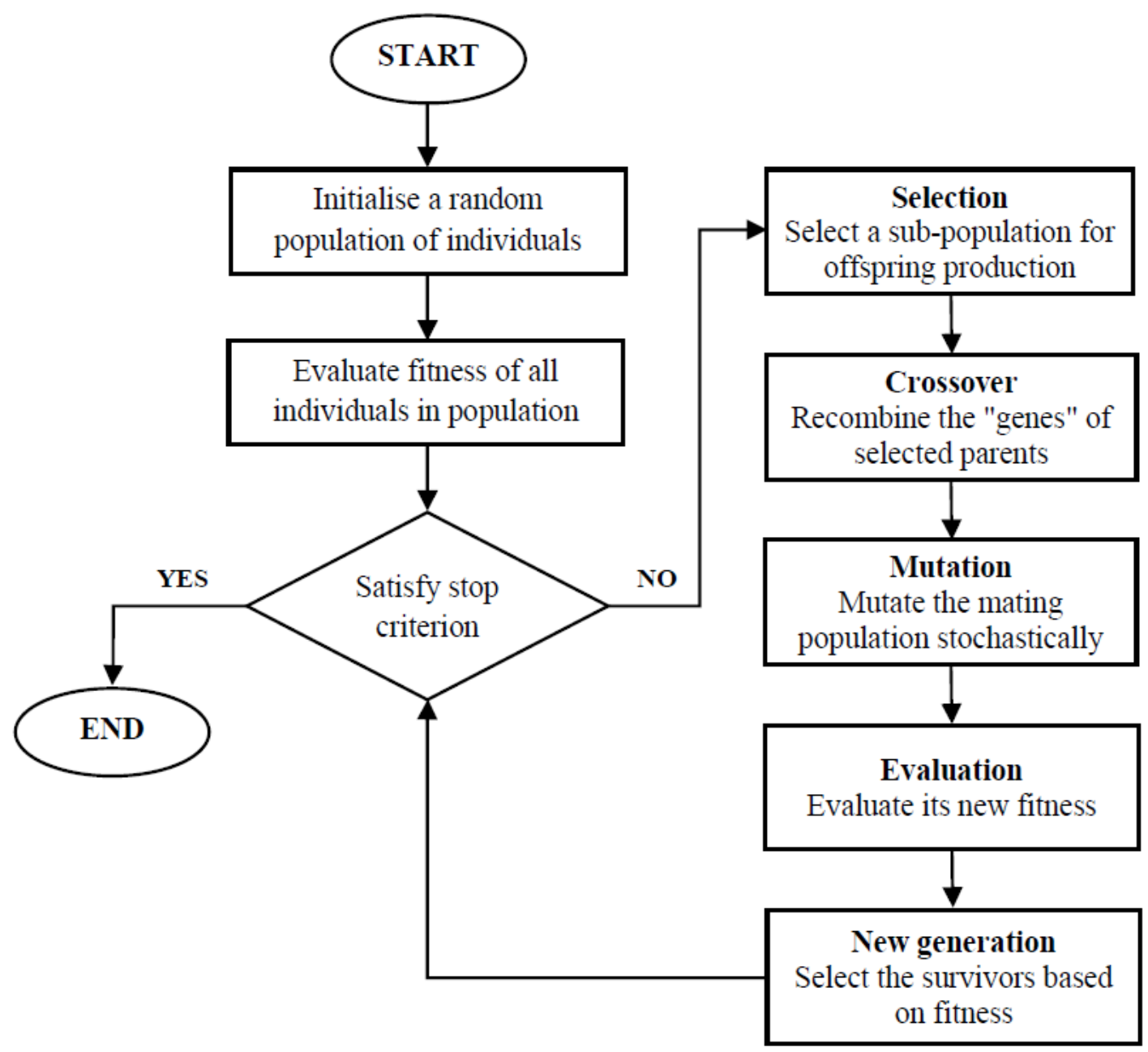

Fig. 8. Genetic Algorithm optimization process

For the sake of comparison, a similar model composed of the platform and a single TMD is set up. By using GA in the Optimization Tool of MATLAB program, optimum parameters can be obtained for both TMD and HTMD. In the optimization procedure, the frequency ratio $(\alpha)$ is still considered from 0.5 to 1.5 and parameters of the offshore platform are taken from Table 1. Variables, constraints and GA's parameters used in the Optimization Tool (MATLAB) are shown in Table 2. 
Table 2. Variables, constraints and GA's parameters used in the Optimization Tool (MATLAB)

\section{Parameters of GA}

\begin{tabular}{lllll}
\multicolumn{2}{c}{ Parameters of GA } & & \multicolumn{2}{c}{ Variables and constraints } \\
\cline { 1 - 2 } Parameter & Chosen value & & Variables & Constraints \\
\cline { 1 - 2 } Number of variables & 6 & & $\mu$ & 0.03 (fixed) \\
Population size & 200 & & $\mu_{3}$ & {$[0,1.0]$} \\
Number of generations & $600\left(100^{*}\right.$ Number of variables $)$ & & $\xi_{2}$ & {$[0,0.5]$} \\
Crossover fraction & 0.8 & & $\xi_{d}$ & {$[0,0.5]$} \\
Elite count & $10\left(0.05^{*}\right.$ Population size $)$ & & $\beta_{1}$ & {$[0.5,1.5]$} \\
Others & Use default values in this tool & $\beta_{2}$ & {$[0.5,1.5]$} \\
\hline
\end{tabular}

The optimal parameters for both HTMD and TMD are reported in Table 3. It can be observed that the optimum damping ratio of the TMD in $\operatorname{HTMD}\left(\xi_{2 o p t}=0.00236\right)$ is extremely small (close to zero). In contrast, the optimum damping ratio of TLCD is much larger than that of TMD $\left(\xi_{\text {dopt }}=\right.$ 0.209 compared with $\left.\xi_{o p t}^{T M D}=0.1099\right)$.

Table 3. Optimal parameters for both HTMD and TMD in the case of $\mu=0.03$

\section{Optimal parameters of HTMD}

\begin{tabular}{lllllllllll}
\hline$\beta_{10 p t}$ & $\beta_{2 o p t}$ & $\mu$ & $\mu_{3 o p t}$ & $\xi_{2 o p t}$ & $\xi_{\text {dopt }}$ & & $\beta_{\text {opt }}^{T M D}$ & $\mu$ & $\xi_{\text {opt }}^{\text {TMD }}$ \\
0.9363 & 1.069 & 0.03 & 0.1955 & 0.00236 & 0.209 & & 0.96 & 0.03 & 0.1099 \\
\hline
\end{tabular}

\section{EFFECTIVENESS AND ROBUSTNESS OF HTMD}

In this section, the effectiveness and robustness of the optimized HTMD are calculated and compared with an optimum TMD having the same weight as HTMD. It is necessary to introduce the following indexes for evaluating the efficiency of both TMD and HTMD. 


\subsection{Evaluation index}

The peak dynamic magnification factor $\left(D M F_{\max }\right)$ of the structural response (Yamaguchi and Harnpornchai, 1993; Gao et al., 1999) and the root mean square of the peak acceleration response $\left(R M S_{A_{1}}\right)$ of the structure (Xue et al., 2016) are two essential indices for measuring the vibration absorption capacity of a damper. It is noted that the absorber, which gives the larger value of $D M F_{\max }$ or $R M S_{A_{1}}$, is less effective (Xue et al., 2016).

As used in the paper of Gao et al. (1999), $D M F_{\max }$ of the structural response on the excitation frequency range of $\left[\Omega_{1}, \Omega_{2}\right]$ (corresponding to $\left[\alpha_{1}, \alpha_{2}\right]$ ) is determined as

$$
D M F_{\text {max }}=\operatorname{Max}\left\{U_{1}\right\}_{\alpha_{2}}^{\alpha_{1}} /\left(F_{0} / K_{1}\right)
$$

Therefore, the $D M F_{\text {max }}$ reduction index of HTMD or TMD can be defined as

$$
\varphi=\frac{D M F_{\max , \text { none }}-D M F_{\max , \text { absorber }}}{D M F_{\max , \text { none }}} \times 100 \%
$$

In Eq. (25), subscripts "absorber" and "none" are denoted for the structural system with and without the absorber, respectively.

The root mean square of the peak acceleration response $\left(R M S_{A_{1}}\right)$ of the platform (Wu et al., 2018; Xue et al., 2016) is given by

$$
R M S_{A_{1}}=\sqrt{\frac{\sum_{1}^{n}\left(A_{1 i}\right)^{2}}{n}}
$$

In Eq. (26), $A_{1 i}$ is the value of peak acceleration response corresponding to $\Omega_{i}$. As a result, the $R M S_{A_{1}}$ reduction index (Wu et al., 2018) for both TMD and HTMD is also calculated by

$$
\theta=\frac{R M S_{A_{1, \text { none }}}-R M S_{A_{1, \text { absorber }}}}{R M S_{A_{1, \text { none }}}} \times 100 \%
$$

Similarly, subscripts "absorber" and "none" are also denoted for the cases with and without the absorber, respectively. 


\subsection{Effectiveness of HTMD}

Table 4 shows the maximum vibration absorption capacity of TMD and HTMD in the case of $\mu=0.03$. Apart from $D M F_{\text {max }}$ and $R M S_{A_{1}}$, other indexes for both the optimized HTMD and TMD are also calculated in Table 4. In the table, the value of $D M F_{\text {max }}$ for the system uncontrolled is 12.5 . Meanwhile, this value is 4.504 for the system with the optimum HTMD and is 5.241 for the case with the optimized TMD. It can be found from Table 4 that all evaluation criteria of HTMD are better those of TMD. This means that the performance of HTMD is higher than that of TMD in suppressing vibration. With the same value of $\mu=0.03$, there is a significant increase of approximately $6 \%$ in the $D M F_{\text {max }}$ reduction index $(\varphi)$ of the system with the HTMD compared with that of the system with the TMD. Nevertheless, the improvement of the $R M S_{A_{1}}$ reduction index $(\theta)$ of the system with the HTMD is not much, only $1.3 \%$.

Table 4. Vibration absorption capacity of optimum HTMD and TMD

\begin{tabular}{lcccc}
\hline Configuration & $\boldsymbol{D} \boldsymbol{M F}_{\text {max }}$ & $\boldsymbol{R} \boldsymbol{M} \boldsymbol{S}_{\boldsymbol{A}_{\mathbf{1}}}$ & $\boldsymbol{\varphi}$ & $\boldsymbol{\theta}$ \\
\hline Non-controlled & 12.5 & 1.399 & $0 \%$ & $0 \%$ \\
With TMD & 5.241 & 1.015 & $58.1 \%$ & $27.4 \%$ \\
With HTMD & 4.504 & 0.997 & $64.0 \%$ & $28.7 \%$ \\
\hline
\end{tabular}

\subsection{Robustness of HTMD}

It is not easy to exactly measure the structural stiffness of a real platform. Hence, there exists an error in determining the natural frequency of the platform (Yamaguchi and Harnpornchai, 1993). In addition, due to different reasons, the platform's stiffness can be changed (Xue et al., 2016). One of these reasons can come from environmental impacts or pieces of equipment replaced on the platform. Thus, to study the robustness of HTMD, the effect of the variation of the platform's stiffness and the drift in the absorber's damping ratio is analysed here. 
It is assumed that the error in the platform's stiffness $\Delta K_{1}$ changes in the range of $[-30 \%, 30 \%]$. Fig. 9 presents the robustness of the optimum HTMD and TMD against the change of the structural stiffness. In Fig. 9, the optimized TMD and the optimum HTMD have the greatest performance at nominal stiffness $\left(\Delta K_{1}=0 \%\right)$. As reported in Table $4, D M F_{\max }$ of the platform response with the optimum TMD and HTMD at $\Delta K_{1}=0 \%$ are 5.241 and 4.504, respectively. Obviously, when the structural stiffness varies, both the optimized TMD and HTMD become non-optimized absorbers and less effective. From the results obtained, it is found that the robustness of the optimum HTMD against the variation in the structural stiffness is not better than that of the optimized single TMD. However, one has many options for both frequency ratio and damping ratio to maintain a similar level of effectiveness as an optimized TMD. In this way, an HTMD is much more robust than a TMD.

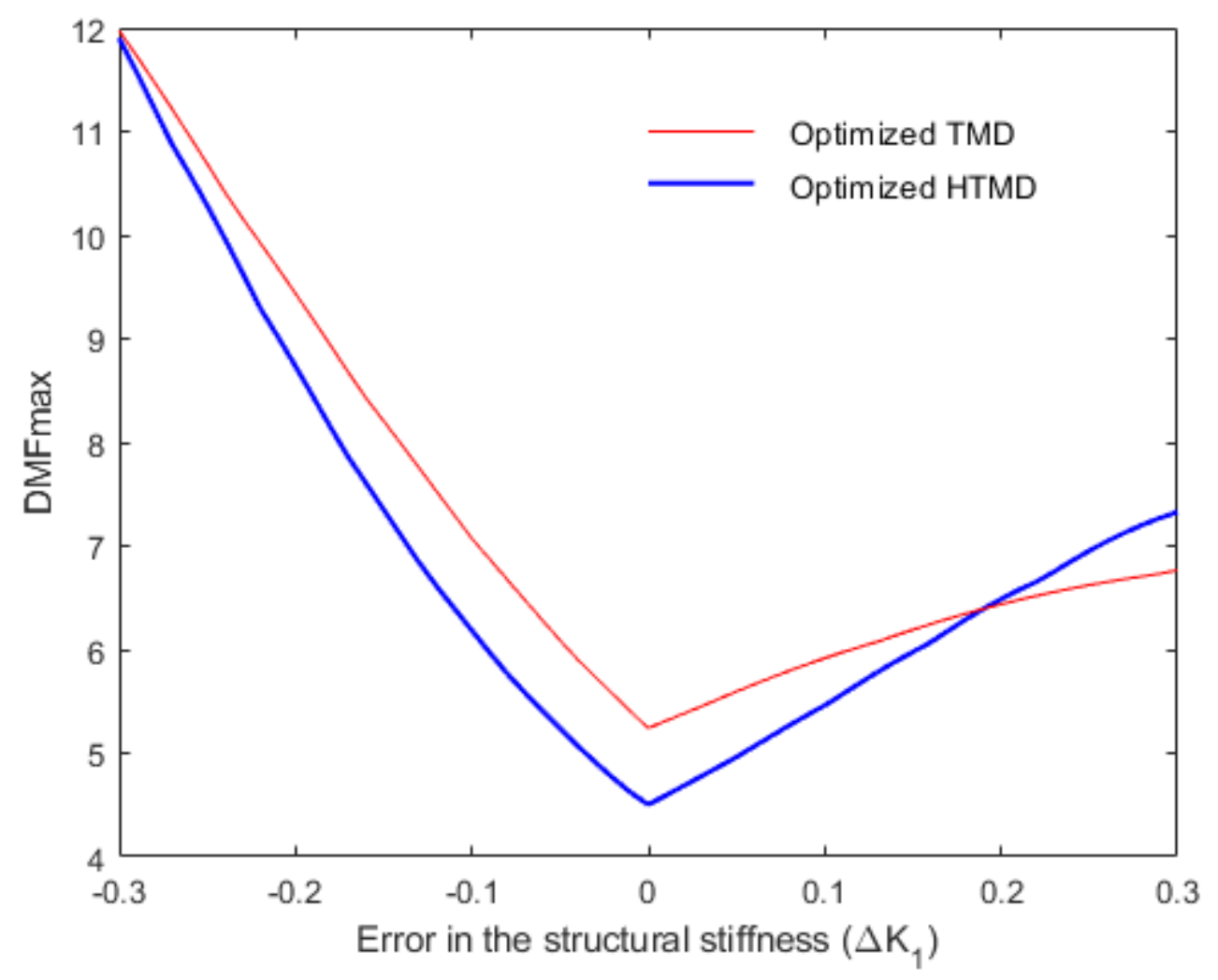

Fig. 9. $D M F_{\max }$ curve with the variation of $\Delta K_{1}$

The robustness of HTMD or TMD against the change of the absorber's damping ratio $\left(\Delta \xi_{2}\right)$ within $[-50 \%, 50 \%]$ is described in Fig. 10. In this case, the drift of the damping ratio $\Delta \xi_{2}$ in the HTMD is 
only considered for the primary damper (TMD in HTMD). In the figure, the variation of the effectiveness of the HTMD based on the value of $D M F_{\max }$ seems to be not significant (4.504 compared with 4.521 as the damping ratio $\xi_{2}$ increases by 50\%). As a result, the HTMD remains at the same efficiency level, although the damping ratio changes in an extensive range of $[-50 \%, 50 \%]$. Conversely, $D M F_{\max }$ of the system with the optimized TMD is increased rapidly due to the change of the damping ratio from the optimum value. In particular, the value of $D M F_{\text {max }}$ rises from 5.241 (at $\left.\Delta \xi_{2}=0\right)$ to 6.201 (at $\Delta \xi_{2}=-50 \%$ ) or to $\left(5.967\right.$ at $\left.\Delta \xi_{2}=50 \%\right)$.

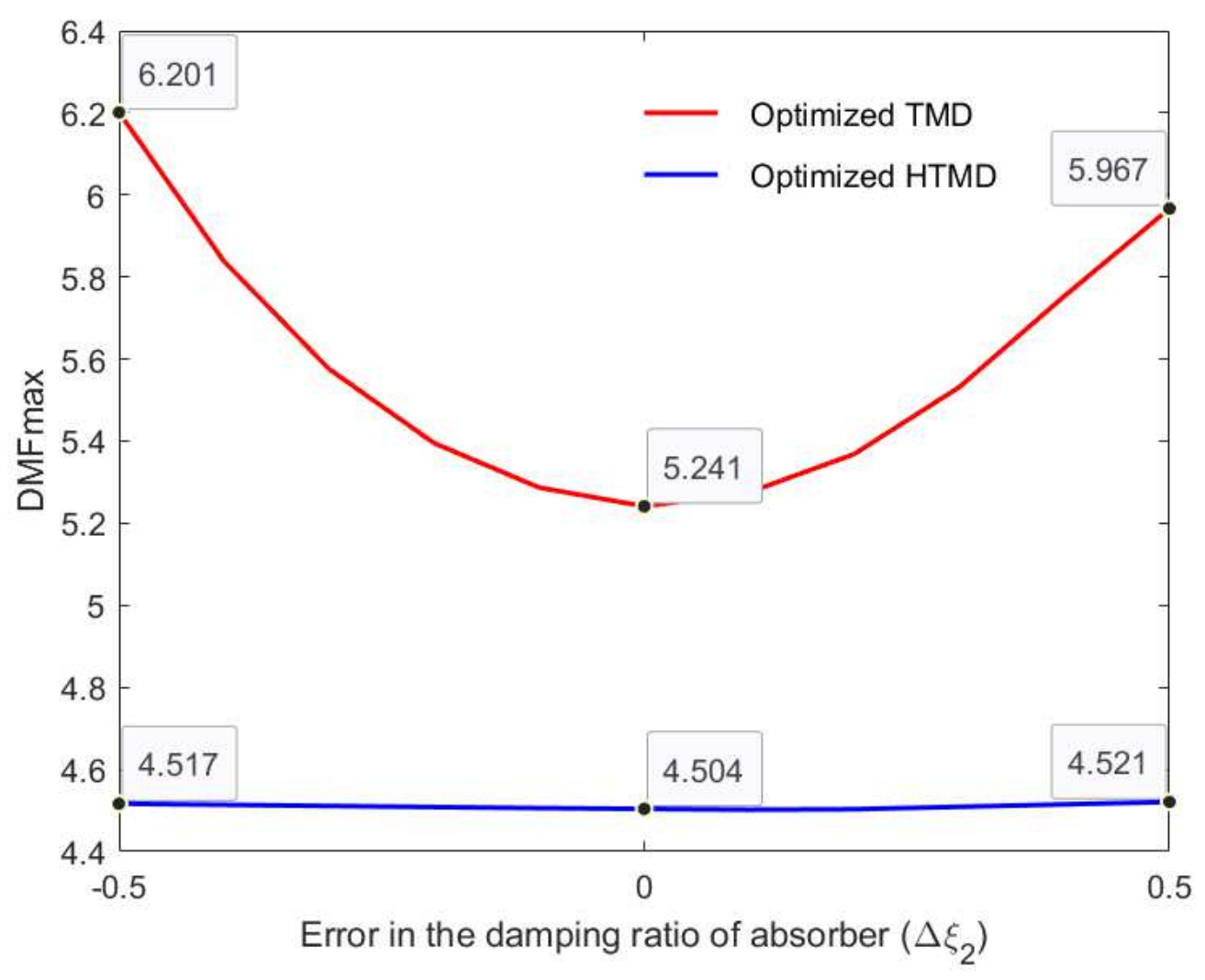

Fig. 10. $D M F_{\max }$ curve with the change of $\Delta \xi_{2}$

\section{MULTI-OBJECTIVE OPTIMAL DESIGN}

The HTMD is the equipment added to the main structure to reduce structural vibrations. As a result, the weight of HTMD is always limited due to the safety of the structure. When using the HTMD to mitigate responses of the platform, there are several main objectives that should be 
considered. For example, they may be the effectiveness, robustness or weight of HTMD. In this study, two core objectives are the performance and weight of HTMD will be discussed.

The aim of the multi-objective optimal design in this paper is to maximize the vibrational absorption capacity of HTMD as well as to minimize the weight of HTMD. Herein, the performance of the damper is evaluated through the value of $D M F_{\max }$ in the excitation frequency range of $\left[\Omega_{1}\right.$, $\Omega_{2}$ ] (corresponding to the frequency ratio from $\alpha_{1}$ to $\alpha_{2}$ ) and the device's weight added to the structure is defined through the nondimensional quantity $\mu$. In this research, $\mu$ is considered in the range of $[1 \%, 5 \%]$. The objective function can be expressed by

$$
\text { Multi_obj }=\left\{\begin{array}{c}
\operatorname{Min}\left\{\left.D M F_{\max }\right|_{\alpha_{2}} ^{\alpha_{1}}\right\} \\
\operatorname{Min}(\mu)
\end{array}\right.
$$

Using GAs in Optimization Tool of MATLAB with the objective functions in Eq. (28), the results are shown in Fig. 11 and Table 5 for both HTMD and TMD.

Table 5 reports the change of $D M F_{\max }$ and $R M S_{A_{1}}$ for different values of the mass ratio. As observed from Table 5, the effectiveness of the optimum HTMD is significantly higher than that of the optimized TMD for each value given of $\mu$. In particular, the effectiveness of the optimized HTMD at $\mu=3 \%$ (as 4.504 ) is better than that of the optimum TMD with $\mu=5 \%$ (as 4.629). Additionally, Table 5 also presents other indexes including $\varphi$ and $\theta$ in both cases with the optimum TMD and with the optimized HTMD as the mass ratio changes. As reported in Table 5, if the mass ratio increases from $1 \%$ to $5 \%$, the performance of the optimized HTMD also increases from $46.5 \%$ to $69.5 \%$ based on the index $\varphi$ and from $17.7 \%$ to $33.9 \%$ based on the index $\theta$. Meanwhile, the effectiveness of the optimum TMD is from $41.9 \%$ to $63 \%$ and from $17.1 \%$ to $33.5 \%$ on the indexes $\varphi$ and $\theta$, respectively. As $\mu$ varies within $[0.01,0.05]$, there is an increase in the index $\varphi$ from $4.6 \%$ to $6.8 \%$ for the oscillation absorption capacity of HTMD compared with those of TMD. The improvement of the index $\theta$ between the HTMD and TMD is not much, only from $0.4 \%$ to $1.3 \%$. 
Table 5. The effectiveness of each damper for different values of $\mu$

\begin{tabular}{|c|c|c|c|c|c|c|c|c|}
\hline \multirow{2}{*}{$\mu$} & \multicolumn{4}{|c|}{ Optimal TMD } & \multicolumn{4}{|c|}{ Optimal HTMD } \\
\hline & $D M F_{\max }$ & $R M S_{A_{1}}$ & $\varphi$ & $\boldsymbol{\theta}$ & $D M F_{\max }$ & $R M S_{A_{1}}$ & $\varphi$ & $\boldsymbol{\theta}$ \\
\hline $1.0 \%$ & 7.266 & 1.160 & $41.9 \%$ & $17.1 \%$ & 6.684 & 1.151 & $46.5 \%$ & $17.7 \%$ \\
\hline $1.5 \%$ & 6.590 & 1.108 & $47.3 \%$ & $20.8 \%$ & 6.151 & 1.100 & $50.8 \%$ & $21.3 \%$ \\
\hline $2.0 \%$ & 6.109 & 1.069 & $51.1 \%$ & $23.6 \%$ & 5.651 & 1.061 & $54.8 \%$ & $24.1 \%$ \\
\hline $2.5 \%$ & 5.773 & 1.039 & $53.8 \%$ & $25.7 \%$ & 5.045 & 1.029 & $59.6 \%$ & $26.4 \%$ \\
\hline $3.0 \%$ & 5.241 & 1.015 & $58.1 \%$ & $27.4 \%$ & 4.504 & 0.997 & $64.0 \%$ & $28.7 \%$ \\
\hline $3.5 \%$ & 5.113 & 0.983 & $59.1 \%$ & $29.7 \%$ & 4.292 & 0.976 & $65.7 \%$ & $30.2 \%$ \\
\hline $4.0 \%$ & 4.976 & 0.965 & $60.2 \%$ & $31.0 \%$ & 4.122 & 0.954 & $67.0 \%$ & $31.8 \%$ \\
\hline $4.5 \%$ & 4.803 & 0.947 & $61.6 \%$ & $32.3 \%$ & 3.968 & 0.940 & $68.3 \%$ & $32.8 \%$ \\
\hline $5.0 \%$ & 4.629 & 0.930 & $63.0 \%$ & $33.5 \%$ & 3.807 & 0.925 & $69.5 \%$ & $33.9 \%$ \\
\hline
\end{tabular}

Fig. 11 describes the Pareto curves for the two-objective optimal design of each damper. It is observed that the effectiveness of absorbers will increase if their mass increases. One chooses to improve the performance of HTMD, then the target in minimizing the weight of HTMD added to the primary structure will be not able. Obviously, there always exists a law of trade-off in the multiobjective optimization problems. For the Pareto curves in Fig. 11, they will help designers can choose a suitable value for both objectives from these Pareto sets instead of considering the full range of every parameter. 


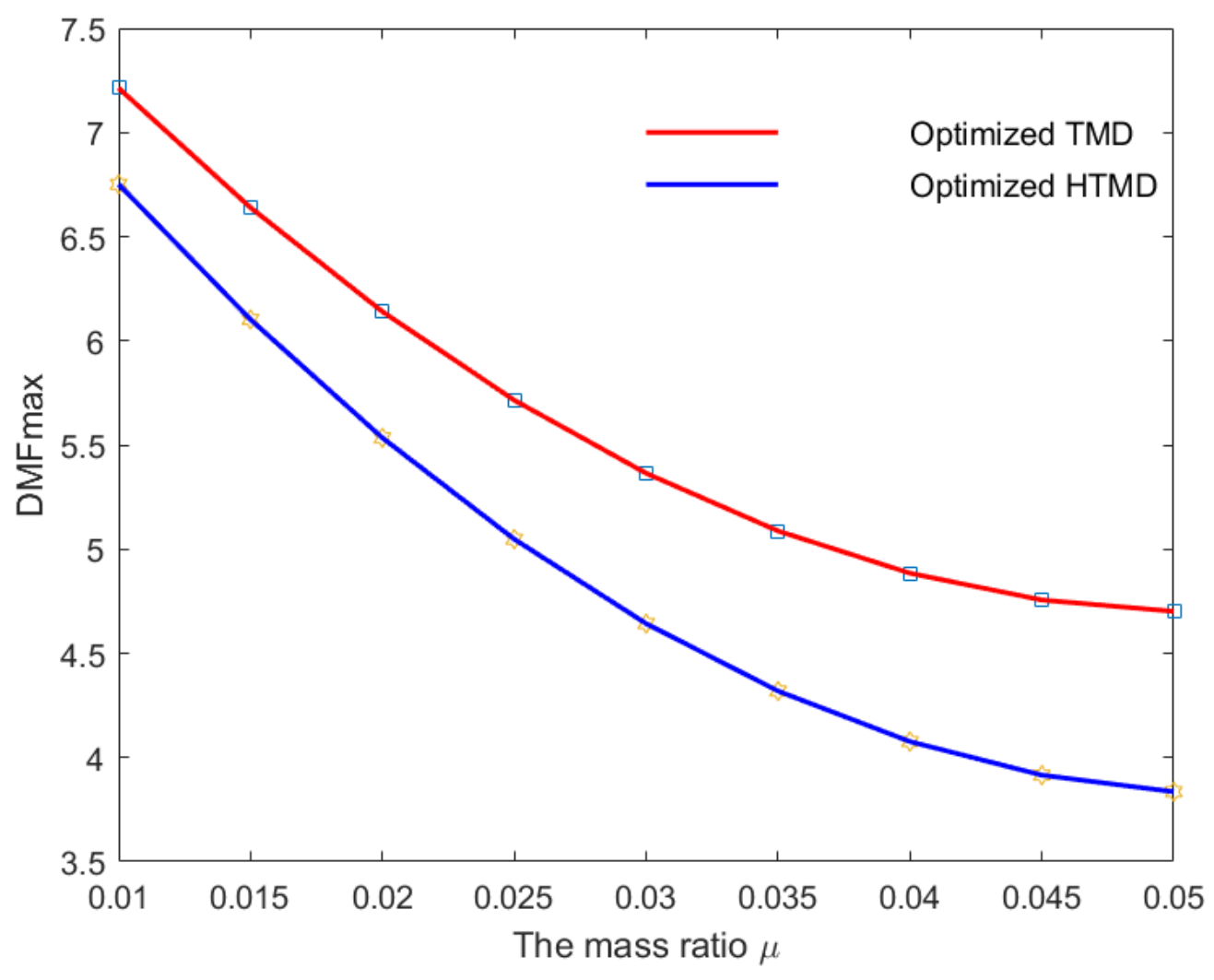

Fig. 11. Pareto curve of each absorber

\section{SUMMARY AND CONCLUSIONS}

In this paper, a hybrid type of TMD was proposed for vibration suppression of the offshore platform under harmonic excitation. First, a parametric study was conducted to explore the critical characteristics of HTMD. It was found that the mass ratio $\mu_{3}$ plays a vital role in the vibration control capacity of HTMD. In particular, an HTMD is effective if the ratio $\mu_{3}<1$. By contrast, the performance of HTMD is insignificant if $\mu_{3} \geq 1$. Then a set of optimal parameters of HTMD was determined through the optimization procedure. The performance and robustness of HTMD were also evaluated and compared with those of an optimum TMD. The obtained results showed that an optimum HTMD offers a higher level of performance in reducing dynamic responses compared with an optimized traditional TMD having the same weight as HTMD. In terms of robustness, the HTMD is not robust in resisting the variation of the structural stiffness. 
The efficiency of HTMD in reducing the undesired vibrations of the platform under the harmonic force was demonstrated. In addition, the numerical computation shows that the optimum damping ratio of the TMD is minimal, close to zero. Obviously, the damping ratio of TMD does not play an essential role in the HTMD. Using an undamped TMD instead of a regular TMD will offer an outstanding advantage in the maintenance process as well as the installation because of its simplified configuration. Therefore, this is why a new device named Upgraded Tuned Liquid Column Damper (UTLCD) has been developed in another paper of the same author (Cao, 2021). Unlike the HTMD introduced in this paper, Cao (2021) proposed the UTLCD based on the combination between an undamped TMD and a single TLCD.

\section{DECLARATIONS}

\section{Funding Acknowledgement:}

This research received no specific grant from any funding agency in the public, commercial, or notfor-profit sectors.

\section{Conflict of interest statement:}

The authors declare no conflict of interest in preparing this article. 


\section{REFERENCES}

Ahadi P, Mohebbi M and Shakeri K (2012) Using optimum multiple tuned liquid column dampers for mitigating the seismic response of structures. ISRN Civil Engineering 2012: Article ID 592181.

Al-Saif KA, Aldakkan KA and Foda MA (2011) Modified liquid column damper for vibration control of structures. International Journal of Mechanical Sciences 53 (2011) 505-512.

Cao QH (2021) Vibration Control of Structures by an Upgraded Tuned Liquid Column Damper. Journal of Engineering Mechanics 2021, 147(9): 04021052

Chang CC and Hsu CT (1998) Control performance of liquid column vibration absorbers. Engineering Structures 20(7): 580-586.

Diana G, Resta F, Sabato D and Tomasini G (2013) Development of a methodology for damping of tall buildings motion using TLCD devices. Wind and Structures 17: 629-646.

Gao H and Kwok KCS (1997) Optimization of tuned liquid column dampers. Engineering Structures 19: 476-486.

Gao H, Kwok KCS and Samali B (1999) Characteristics of multiple tuned liquid column dampers in suppressing structural vibration. Engineering Structures 21: 316-331.

Gil-Martín LM, Carbonell-Márquez JF, Hernández-Montes E, Aschheim M and Pasadas-Fernández M (2012) Dynamic magnification factors of SDOF oscillators under harmonic loading. Applied Mathematics Letters 25: 38-42.

Ikago K, Saito K and Inoue N (2012) Seismic control of single-degree-of-freedom structure using tuned viscous mass damper. Earthquake Engineering and Structural Dynamics 41: 453-474.

Jafarabad A, Kashani M, Parvar MRA and Golafshani AA (2014) Hybrid damping systems in offshore jacket platforms with float-over deck. Journal of Constructional Steel Research 98 (2014): 178-187.

Li C and Zhu B (2006) Estimating double tuned mass dampers for structures under ground acceleration using a novel optimum criterion. Journal of Sound and Vibration 298: 280-297. 
McCall J (2005) Genetic algorithms for modelling and optimisation. Journal of Computational and Applied Mathematics 184: 205-222.

Momtaz AA, Abdollahian MA and Farshidianfar A (2017) Study of wind-induced vibrations in tall buildings with tuned mass dampers taking into account vortices effects. International Journal Advanced Structural Engineering 9: 385-395.

Ou J, Long X, Li QS and Xiao YQ (2007) Vibration control of steel jacket offshore platform structures with damping isolation systems. Engineering Structures 29: 1525-1538.

Samali B, Mayol E, Kwok KCS, Mack A and Hitchcock P (2004) Vibration control of the windexcited 76-story Benchmark Building by liquid column vibration absorbers. Journal of Engineering Mechanics 130: 478-485.

Vellar LS, Ontiveros-Pérez SP and Miguel LFF (2019) Robust optimum design of multiple tuned mass dampers for vibration control in buildings subjected to seismic excitation. Shock and Vibration 2019: Article ID 9273714.

Wu J-C, Shih M-H, Lin Y-Y and Shen Y-C (2005) Design guidelines for tuned liquid column damper for structures responding to wind. Engineering Structures 27: 1893-1905.

Wu Q, Zhao W, Zhu W, Zheng R and Zhao X (2018) A tuned mass damper with nonlinear magnetic force for vibration suppression with wide frequency range of offshore platform under earthquake loads. Shock and Vibration 2018: Article ID 1505061.

Wu Q, Zhao X, Zheng R and Minagawa K (2016) High response performance of a tuned-mass damper for vibration suppression of offshore platform under earthquake loads. Shock and Vibration 2016: Article ID 7383679.

Xue Q, Zhang J, He J and Zhang C (2016) Control performance and robustness of pounding tuned mass damper for vibration reduction in sdof structure. Shock and Vibration 2016: Article ID 8021690.

Yalla SK and Kareem A (2000) Optimum absorber parameters for tuned liquid column dampers. Journal of Structural Engineering 126: 906-915. 
Yamaguchi $\mathrm{H}$ and Harnpornchai N (1993) Fundamental characteristics of multiple tuned mass dampers for suppressing harmonically forced oscillations. Earthquake Engineering and Structural Dynamics 22: 51-62. 